\title{
A novel Acoustic Fluid Level Estimation Method Based on Evidence Fusion Mechanism
}

\author{
Zhenghui Li ${ }^{1, *}$, Na Zhang ${ }^{1}$, Feiya Lv $^{2}$, Jingya Yang ${ }^{1}$ \\ ${ }^{I}$ School of Electrical Engineering, Zhengzhou Railway Vocational \& Technical College, Zhengzhou, \\ Henan, China \\ ${ }^{2}$ School of software, Anyang Normal University, Anyang, Henan, China \\ *Corresponding Author.
}

\begin{abstract}
In order to reduce the missed detection error and the systematic error caused by acoustic resonance fluid level detection, liquid level estimation method based on evidence fusion mechanism is designed. It establishes a two dimensional dynamic system model of the standing wavelength. The state evidence of wavelength is obtained through the random set description of evidence, and the extension principle of random set is used to get the observation evidence of wavelength. The evidential reasoning (ER) rule and dependent evidence fusion are used to fuse those evidence, and the estimation value of fluid level can be calculated from fused result based on pignistic expectation. The corresponding liquid level estimation experiment illustrates the validity and feasibility of the proposed method.
\end{abstract}

Keywords: Fluid level estimation, evidential reasoning, dependent evidence fusion

\section{Introduction}

The acoustic fluid level detection method play an important role in the production, processing, transportation, reserve calculation, state and alarm monitoring of modern industry system ${ }^{[1]}$. It is widely used in petroleum, chemical industry, sewage treatment and other related fields. Most of these methods are operated in ultrasound region. However, barriers (such as bubbles, ripples and residues) often exist on liquid surface in the actual measurement environment, parasitic reflection phenomena caused by barriers will change ultrasound path, which limit the accuracy of ultrasonic measurement.

Ref ${ }^{[2]}$ presents a fluid level detection method based on low-frequency acoustic resonance. Due to the long wave length of low frequency sound wave, the diffraction phenomenon will occur when the sound wave encounter barriers, which can effectively avoid the measurement inaccuracy caused by parasitic reflection phenomenon. However, its measurement range $(\leq 8.28 \mathrm{~m})$ is limited by the initial resonance frequency (RF) and the sensitivity of receiving sound wave (microphone). $\operatorname{Ref}^{[3]}$ presents a liquid level measurement method based on fixed frequency range acoustic resonance principle, which uses a series of resonance frequencies in fixed frequency range (from $1000 \mathrm{~Hz}$ to $2500 \mathrm{~Hz}$ ) to replace the single initial RF. This method not only expands the measurement range by overcoming the problem of microphone sensitivity, but also improves the real-time performance of measurement.

For improving the accuracy of measurement, ref ${ }^{[4]}$ presents a liquid level error compensation method based on belief rule base. By analyzing the principle of acoustic resonance measurement, it is concluded that the principle error mainly comes from two aspects: 1.The error introduced by the height calculation formula approximately derived from the synthetic acoustic wave; 2.The influence of different sweep duration and sampling frequency on the measurement accuracy. Based on this, the compensation model of measuring height and three parameters is established, which makes the compensator learn the principle error compensation value corresponding to the measured value. However, in the industrial application environment, the detection system is easily affected by the

ISSN: 0010-8189

(C) CONVERTER 2020

www.converter-magazine.info 
complex external noise, and the energy attenuation of sound wave will occur in the transmission process, which will cause the acquisition of some resonance points is missing and inaccurate. The missed detection will cause the extraction bias between the collected resonance point and its true value. In addition, the inherent errors of the speaker, microphone and temperature sensor are transmitted with the conversion formula, resulting in the final detection error. The error caused by transmit/receive acoustic wave system in acoustic resonance level gauge, which can be called systematic error. These problems have seriously affected the anti-interference ability and the accuracy of acoustic resonance method, thus limiting its wide application in various fields.

In response to the above-mentioned problems, this paper presents a novel acoustic fluid level estimation method based on evidence fusion mechanism. Firstly, it establishes a two dimensional dynamic system model based on the relationship between any three adjacent resonance points, which can estimate the missed resonance point and make up for the inaccurate resonance point. Secondly, the state/observation noise of systematic error can be approximated to triangle possibility distribution respectively. It constructs the state evidence and observation evidence by adding those noise via the random set description. Thirdly, the recursive algorithm based on evidence fusion mechanism is proposed to estimate the wavelength of resonance point at each moment. The ER rule and dependent evidence fusion are used to fuse those evidence of recursive algorithm. Finally, the detected height can be calculated from the fused result based on pignistic expectation.

\section{A novel detection method based on two dimensional dynamic system model}

\subsection{The detection principle based on fixed frequency range acoustic resonance}

The structure of level gauge is shown in Figure 1, including a controller, a sound guide tube, a temperature sensor, a sound emission device, a sound receiving device, etc. The sound emitting/receiving devices are cheap speaker and microphone respectively. The sound guide tube is inserted into the liquid to be measured perpendicular to the liquid surface. The microphone, speaker and temperature sensor are installed at one end of the sound guide tube, and the microphone and the speaker are on the same horizontal plane. The other end of the sound guide tube is below the liquid surface, and the detected height is the distance between the plane of the speaker/microphone and the liquid surface. The speaker is perpendicular to the measured liquid surface and continuously emits lowfrequency sound waves in a fixed frequency range. The sound wave reflected by liquid surface and the emitted sound wave are synthesized into synthesis wave in sound guide tube. The emitted/reflected sound wave is expressed as $y_{1} / y_{2}$, respectively.

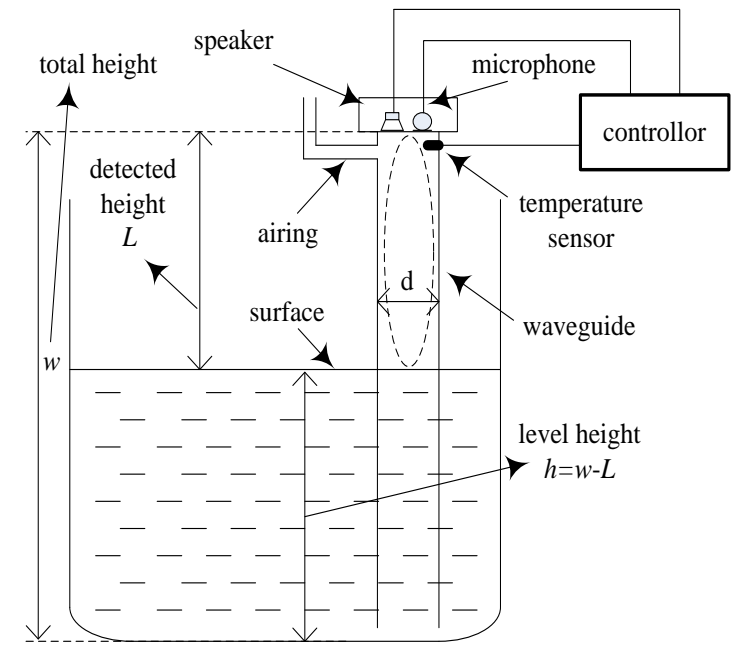

Fig 1: Structure of level gauge

$$
y_{1}=A \cos 2 \pi\left(P t-\frac{L}{\lambda}\right)
$$

ISSN: 0010-8189 


$$
y_{2}=A \cos 2 \pi\left(P t+\frac{L}{\lambda}\right)
$$

Synthesis wave $y$ is derived as

$$
y=2 A \cos \left(\pi \frac{2 L}{\lambda}\right) \cos (2 \pi P t)
$$

where $A$ denotes the amplitude, $\lambda$ and $P$ denote the wavelength and frequency respectively, and $L$ denotes detected height. When the amplitude of synthesis sound wave is the maximum, the relationship between $L$ and $\lambda$ should meet the following formula

$$
L=n(k) \frac{\lambda(k)}{2} \quad k=1,2,3 \cdots
$$

where $\lambda(k)$ denotes the wavelength of standing waves, $k$ denotes the $k^{\text {th }}$ standing waves. $\lambda(k)$ can be calculated as

$$
\lambda(k)=\frac{c}{f(k)}
$$

Where $f(k)$ denotes the corresponding resonance frequency of standing waves. The sound velocity $c=331.4+0.6 T$ and $T$ is temperature in detection environment.

As a result, ref[2] take (5) into (4) and get:

$$
L=\frac{n(k)(331.4+0.6 T)}{2 f(k)}
$$

where, $n(k)$ is given as :

$$
n(k)=f(k) /(f(k+1)-f(k))
$$

and

$$
n(k+1)=n(k)+1
$$

Here, in order to ensure that $n(k)$ is a positive integer, (6) can be expressed as

$$
L=\|n(k)\| c / 2 f(k)
$$

The height of the fluid level can be calculated as

where

$$
h=w-\bar{L}
$$

$$
\bar{L}=\left(\sum_{k=1}^{M-1}(\|n(k)\| c / 2 f(k))\right) /(M-1)
$$

is the mean of $M-1$ times measurements.

Obviously, from (11), it can be seen that the accuracy of this liquid level detection method mainly depends on the accurate acquisition of $f(k)$ or $\lambda(k)$. However, in the industrial application environment, the detection system is easily affected by the complex external noise, and the energy attenuation of sound wave will occur in the transmission process, which will cause the acquisition of some resonance points is missing and inaccurate.In addition, the systematic error (accuracy difference of various sensors in transmit/receive acoustic wave system) will also cause the extraction bias between the collected resonance point and its true value. The above problems have seriously affected the accuracy and anti-interference ability of this method, thus limiting its wide application in various fields.

2.2 Two dimensional dynamic system model of the wavelength under bounded noises

In the new method, in the process of acquiring the resonance points in the synthetic wave, it is no longer limited to extracting the detected resonance points in observation domain. Instead, each resonance point is estimated by a dynamic system model based on the relationship between any three adjacent resonance points, which can make up

ISSN: 0010-8189

(C) CONVERTER 2020

Www.converter-magazine.info 
for the missing resonance points and compensate for the inaccurate resonance points. At the same time, by constructing and adding observation noise and state noise to the dynamic system model, the anti-interference ability of new method can be effectively enhanced. From(4), obviously, we can get a set of equations

$$
\begin{gathered}
L=n(k-1) \frac{\lambda(k-1)}{2} \\
L=n(k) \frac{\lambda(k)}{2} \\
L=n(k+1) \frac{\lambda(k+1)}{2}
\end{gathered}
$$

Where $\lambda(k-1), \lambda(k)$ and $\lambda(k+1)$ denote the wavelength of the three adjacent resonance points in the standing waves respectively. Substituting (12) into (13), we obtain

$$
L=\frac{\lambda(k-1) \lambda(k)}{2[\lambda(k-1)-\lambda(k)]}
$$

From(15), the value of detected height $L$ can be obtained by any two adjacent wavelengths. In addition, the dynamic equation of wavelength can be obtained in the following forms through the simultaneous (12), (13) and (14)

$$
\lambda(k+1)=\frac{\lambda(k-1) \lambda(k)}{2 \lambda(k-1)-\lambda(k)}
$$

Equation(16) is further reduced to the first-order nonlinear difference form:

$$
\left[\begin{array}{l}
\lambda(k) \\
\lambda(k+1)
\end{array}\right]=\left[\begin{array}{c}
\lambda(k) \\
\frac{\lambda(k-1) \lambda(k)}{2 \lambda(k-1)-\lambda(k)}
\end{array}\right]
$$

By transforming equation (16) into equation (17), a new vector is introduced, which contains two original state vectors. The dynamic systems model is expressed as follows

$$
\left\{\begin{array}{l}
x(k+1)=f[x(k), v(k)] \\
z(k+1)=g[x(k+1), w(k+1)]
\end{array} k=1,2,3 \ldots .\right.
$$

Where $f$ describes the function relation of $x(k+1)$ and $x(k) . g$ describes the unction relation of $x(k+1)$ and $z(k+1)$. $x(k+1)$ is expressed as

$$
x(k+1)=\left[\begin{array}{l}
\lambda(k+1) \\
\lambda(k+2)
\end{array}\right]
$$

And the state equation of dynamic systems modeled can be expressed as

$$
\left[\begin{array}{c}
\lambda(k+1) \\
\lambda(k+2)
\end{array}\right]=\left[\begin{array}{c}
\lambda(k+1) \\
\frac{\lambda(k) \lambda(k+1)}{2 \lambda(k)-\lambda(k+1)}
\end{array}\right]+\left[\begin{array}{c}
v(k) \\
v(k+1)
\end{array}\right]
$$

Similarly, the observation equation of dynamic systems modeled can be expressed as

ISSN: 0010-8189 


$$
z(k+1)=\left[\begin{array}{c}
\lambda(k+1) \\
\lambda(k+2)
\end{array}\right]+\left[\begin{array}{c}
w(k+1) \\
w(k+2)
\end{array}\right]
$$

$v(k)$ denote $k^{\text {th }}$ state noise, and $w(k)$ denote $k^{\text {th }}$ observation noise. These two noises are independent and identically distributed bounded noise.

\section{Foundations of evidence fusion mechanism}

The main concept and theory of evidence fusion mechanism are presented in section 3.1 and 3.2. There are more detailed theoretical explanations and applications in ref ${ }^{[5-7]}$.

\subsection{Evidential reasoning(ER) theory}

The research object of ER rules is the element and subset in the discernment frame $\Theta=\left\{h_{1}, \ldots, h_{\mathrm{N}}\right\}$. $\Theta$ contains all the possibilities, and the proposition (or element) is mutually exclusive in $\Theta$. $2^{\Theta}$ denote the power set of $\Theta$.The evidence can be expressed as ${ }^{[5-6]}$

$$
e_{j}=\left\{\left(\theta, p_{\theta, j}\right), \forall \theta \subseteq \Theta, \sum_{\theta \subseteq \Theta} p_{\theta, j}=1\right\}
$$

Where $p^{\theta, \mathrm{j}}$ indicates degree of belief for this evidence. $\left(\theta, p^{\theta, \mathrm{j}}\right)$ is an element of $e_{\mathrm{j}}$, and $\theta$ is any subset of $\Theta$ except for the empty set. $\left(\theta, p^{\theta, \mathrm{j}}\right)$ is referred to as a focal element of $e_{\mathrm{j}}$ if $p^{\theta, \mathrm{j}}>0 . r_{\mathrm{j}}$ and $w_{\mathrm{j}}$ represent the reliability and importance of a piece of evidence respectively. $r_{\mathrm{j}}$ describes ability to provide accurate information for a given problem, and reflects the inherent characteristics of the information source. $w_{\mathrm{j}}$ depends on other fused evidence and the specific occasions of application. The belief distribution including $r_{\mathrm{j}}$ and $w_{\mathrm{j}}$ can be expressed as

$$
m_{j}=\left\{\left(\theta, \tilde{m}_{\theta, j}\right), \forall \theta \subseteq \Theta ;\left(P(\Theta), \tilde{m}_{P(\Theta), j}\right)\right\}
$$

Where $\tilde{m}_{\theta, j}$ measures the degree of support for $\theta$ from $e_{\mathrm{j}}$, defined as follows

$$
\tilde{m}_{\theta, j}= \begin{cases}0 & \theta=\varnothing \\ c_{r w, j} m_{\theta, j} & \theta \subseteq \Theta, \theta \neq \varnothing \\ c_{r w, j}\left(1-r_{j}\right) & \theta=P(\Theta)\end{cases}
$$

Where $m_{\theta, j}=w_{j} p_{\theta, j} \cdot c_{r w, j}=1 /\left(1+w_{j}-r_{j}\right)$ is uniquely determined to satisfy $\sum_{\theta \subseteq \Theta} \tilde{m}_{\theta, j}+\tilde{m}_{P(\Theta), j}=1$. ER fusion rule is defined as

$$
\begin{aligned}
p_{\theta, e(2)}= & \begin{cases}0 & \theta=\varnothing \\
\frac{\hat{m}_{\theta, e(2)}}{\sum_{D \subseteq \Theta} \hat{m}_{D, e(2)}} & \theta \subseteq \Theta, \theta \neq \varnothing\end{cases} \\
\hat{m}_{\theta, e(2)}= & {\left[\left(1-r_{1}\right) m_{\theta, 1}+\left(1-r_{0}\right) m_{\theta, 2}\right] } \\
& +\sum_{B \cap C=\theta} m_{B, 1} m_{C, 2} \quad \forall \theta \subseteq \Theta
\end{aligned}
$$

Where $p_{\theta, e(2)}$ is the fusion belief function. It represents the combined belief degree of independent evidence $e_{1}$ and $e_{2}$ support for the proposition $\theta$.

\subsection{Basic concepts in dependent evidence fusion}

In ER Theory, it requires two pieces of evidence to be independent of each other, but evidence dependence often exist in practice. Ref[8] pointed out that if the evidences are derived from multiple independent information sources, when different evidence uses the same information source, these evidences are often mutually dependent.The energy $\operatorname{En}(E)$ is expressed as: 


$$
\operatorname{En}(E)=\sum_{\substack{i=1 \\ A_{i} \neq \Theta}}^{n(E)} \frac{m\left(A_{i}\right)}{\left|A_{i}\right|}
$$

where $\left|A_{\mathrm{i}}\right|$ is the number of elements, $n(E)$ is the number of focal elements contained in evidence $E$. $\operatorname{En}(E)=0$ or $\operatorname{En}(E)=1$ means the smallest or the largest, respectively.

For evidence $E_{1}=\left(\mathrm{F}_{1}, m_{1}\right)$ and evidence $E_{2}=\left(\mathrm{F}_{2}, m_{2}\right)$ defined on $\Theta$, if $E_{1}$ and $E_{2}$ are derived from the same independent information, the energy of the intersection part between $E_{1}$ and $E_{2}$ can be defined as

$$
\operatorname{En}\left(E_{1}, E_{2}\right)=\sum_{\substack{i j=1 \\ D_{i j} \neq \varnothing}}^{\left|\left\{D_{i j}\right\}\right|} \frac{m\left(D_{i j}\right)}{\left|D_{i j}\right|}
$$

Where $D_{\mathrm{ij}}$ denotes intersection focal element, $\left|\left\{D_{\mathrm{ij}}\right\}\right|$ is the number of $D_{\mathrm{ij}}$. The degree dependent of $E_{1}$ and $E_{2}$ is shown as

$$
D\left(E_{1}, E_{2}\right)=\frac{2 \operatorname{En}\left(E_{1}, E_{2}\right)}{\operatorname{En}\left(E_{1}\right)+\operatorname{En}\left(E_{2}\right)}
$$

The energy of $E_{1}$ and $E_{2}$ can be distributed proportionally. The energy $\operatorname{En} f\left(E_{1}\right)$ and $\operatorname{En} f\left(E_{2}\right)$ can be calculated as

$$
\begin{gathered}
\operatorname{En}_{f}\left(E_{1}\right)=\operatorname{En}\left(E_{1}\right)\left(1-\frac{1}{2} D\left(E_{1}, E_{2}\right) * \frac{\operatorname{En}\left(E_{2}\right)}{\operatorname{En}\left(E_{1}\right)}\right) \\
\operatorname{En}_{f}\left(E_{2}\right)=\operatorname{En}\left(E_{2}\right)\left(1-\frac{1}{2} D\left(E_{1}, E_{2}\right) * \frac{\operatorname{En}\left(E_{1}\right)}{\operatorname{En}\left(E_{2}\right)}\right)
\end{gathered}
$$

The dependency coefficient of two evidences is defined as

$$
\begin{aligned}
& R_{12}=\frac{1}{2} D\left(E_{1}, E_{2}\right) \frac{\operatorname{En}\left(E_{2}\right)}{\operatorname{En}\left(E_{1}\right)} \\
& R_{21}=\frac{1}{2} D\left(E_{1}, E_{2}\right) \frac{\operatorname{En}\left(E_{1}\right)}{\operatorname{En}\left(E_{2}\right)}
\end{aligned}
$$

The independent evidence corresponding to $E_{1}$ and $E_{2}$ can be expressed as $E_{1}$ 'and $E_{2}{ }^{\prime}$, BBA functions of $E_{1}{ }^{\prime}$ and $E_{2}{ }^{\prime}$ are shown as:

$$
\begin{aligned}
& m_{1}^{\prime}(A)= \begin{cases}\left(1-R_{12}\right) m_{1}(A), & \forall A \subseteq \Theta, A \neq \Theta \\
1-\sum_{A \subset \Theta} m_{1}^{\prime}(A) & A=\Theta\end{cases} \\
& m_{2}^{\prime}(B)= \begin{cases}\left(1-R_{21}\right) m_{2}(B), & \forall B \subseteq \Theta, B \neq \Theta \\
1-\sum_{B \subset \Theta} m_{2}^{\prime}(B) & B=\Theta\end{cases}
\end{aligned}
$$

The ER rule is used to fuse two evidences ( $E_{1}$ and $E_{2}$ ), so as to realize dependent evidence fusion.

\section{Fluid Estimation based on ER Rule and Dependent Evidence Fusion}

Figure 2 shows the flowchart of the proposed fusion mechanism. The process is described in detail in 6 steps. 


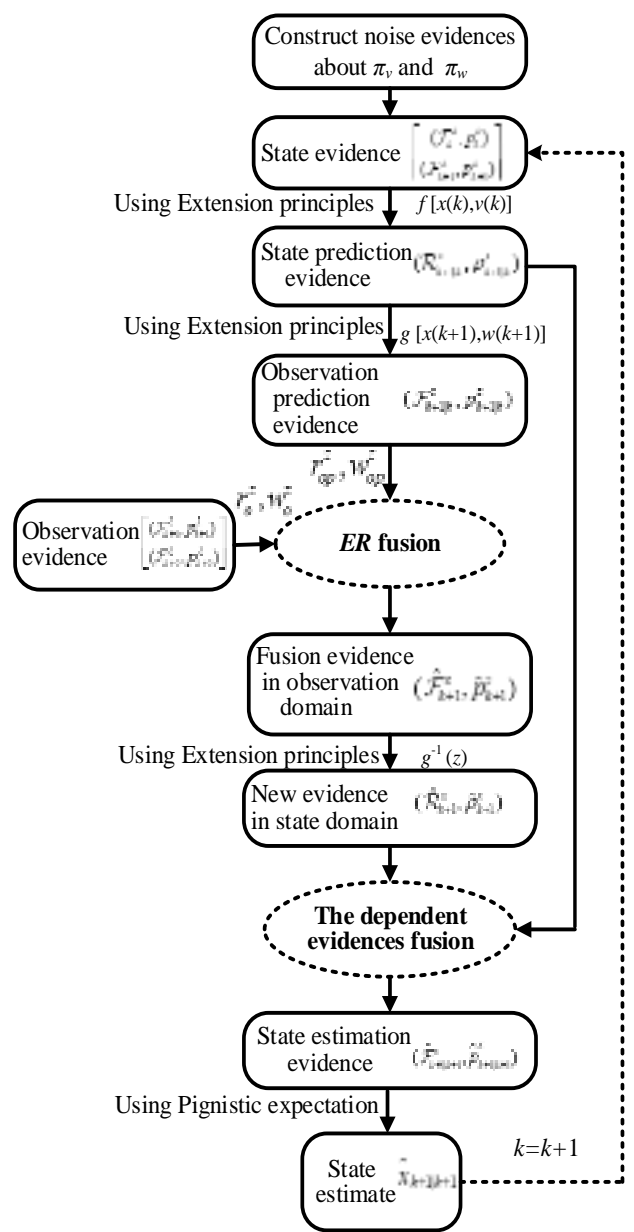

Fig 2: The flowchart of the recursive fusion mechanism

4.1 Construct noise evidence

Construct state noise $v(k)$ and observation noise $w(k)$ into evidence $\left(\mathrm{F}_{k}^{v}, p_{k}^{v}\right)$ and $\left(\mathrm{F}_{k}^{w}, p_{k}^{w}\right) \cdot \pi_{v}$ and $\pi_{w}$ denote approximate the possibility distribution of $v(k)$ and $w(k)$ respectively. For any $\alpha \in(0,1], \alpha$ cut set of $\pi_{v}$ is

$$
\left[\pi_{\alpha}^{v-}, \pi_{\alpha}^{v+}\right]=\left\{v \mid \pi_{v}(v) \geq \alpha\right\}
$$

The corresponding belief distribution can be expressed as

$$
p\left(\left[\pi_{\alpha_{i}}^{v-}, \pi_{\alpha_{i}}^{v+}\right]\right)=\left\{\begin{array}{cc}
\alpha_{i+1} & \text { if } i=0 \\
\alpha_{i+1}-\alpha_{i} & \text { if } i=1,2, \cdots, q-2 \\
1-\alpha_{i} & \text { if } i=q-1
\end{array}\right.
$$

The obtained evidence of $v$ at each moment is the same. For the convenience of subsequent calculations, it is expressed as $\left(\mathrm{F}_{k}^{v}, p_{k}^{v}\right)=\left[\left(\mathrm{F}_{k}^{v 1}, p_{k}^{v 1}\right),\left(\mathrm{F}_{k+1}^{v 2}, p_{k+1}^{v 2}\right)\right]^{T}$ where

$$
\begin{aligned}
& \mathrm{F}_{k}^{v 1}=\left\{\left[\pi_{\alpha_{0}}^{v 1-}, \pi_{\alpha_{0}}^{v 1+}\right],\left[\pi_{a_{1}}^{v 1-}, \pi_{a_{1}}^{v 1+}\right], \cdots,\left[\pi_{\alpha_{q-1}}^{v 1-}, \pi_{\alpha_{q-1}}^{v 1+}\right], \Theta\right\} ; p_{k}^{v 1} \\
& \mathrm{~F}_{k+1}^{v 2}=\left\{\left[\pi_{\alpha_{0}}^{\nu 2-}, \pi_{\alpha_{0}}^{\nu 2+}\right],\left[\pi_{a_{1}}^{\nu 2-}, \pi_{a_{1}}^{v 2+}\right], \cdots,\left[\pi_{\alpha_{q-1}}^{\nu 2-}, \pi_{\alpha_{q-1}}^{\nu 2+}\right], \Theta\right\} p_{k+1}^{v 2} .
\end{aligned}
$$

The noise evidence can be shown as

$$
\left(\mathrm{F}_{k}{ }^{w}, p_{k}^{w}\right)=\left[\left(\mathrm{F}_{k}^{w 1}, p_{k}^{w 1}\right),\left(\mathrm{F}_{k+1}^{w 2}, p_{k+1}^{w 2}\right)\right]^{T} .
$$

4.2 Obtain state prediction evidence at time $k+1$

ISSN: 0010-8189

(C) CONVERTER 2020

Www.converter-magazine.info 
The state evidence $\left(\mathrm{F}_{k}^{\mathrm{x}}, p_{k}^{x}\right)$ of $x_{k \mid k}$ can be obtained by adding noise to $x_{k \mid k}$.

$$
\begin{gathered}
\mathrm{F}_{k}^{\lambda}=\left\{\left[\pi_{\alpha_{0}}^{v-}+\lambda_{k \mid k}, \pi_{\alpha_{0}}^{v+}+\lambda_{k \mid k}\right],\left[\pi_{a_{1}}^{v-}+\lambda_{k \mid k}, \pi_{a_{1}}^{v+}+\lambda_{k \mid k}\right],\right. \\
\left.\cdots,\left[\pi_{\alpha_{q-1}}^{v-}+\lambda_{k \mid k}, \pi_{\alpha_{q-1}}^{v+}+\lambda_{k \mid k}\right], \Theta\right\} ; \quad p_{k}^{\lambda}=p_{k}^{v} \\
\mathrm{~F}_{k+1}^{\lambda}=\left\{\left[\pi_{\alpha_{0}}^{v-}+\lambda_{k+1 \mid k+1}, \pi_{\alpha_{0}}^{v+}+\lambda_{k+1 \mid k+1}\right],\left[\pi_{a_{1}}^{v-}+\lambda_{k+1 \mid k+1}, \pi_{a_{1}}^{v+}+\lambda_{k+1 \mid k+1}\right],\right. \\
\left.\cdots,\left[\pi_{\alpha_{q-1}}^{v-}+\lambda_{k+1 \mid k+1}, \pi_{\alpha_{q-1}}^{v+}+\lambda_{k+1 \mid k+1}\right], \Theta\right\} ; \quad p_{k+1}^{\lambda}=p_{k}^{v} \\
\left(\mathrm{~F}_{k}^{x}, p_{k}^{x}\right)=\left[\begin{array}{c}
\left(\mathrm{F}_{k}^{\lambda}, p_{k}^{\lambda}\right) \\
\left(\mathrm{F}_{k+1}^{\lambda}, p_{k+1}^{\lambda}\right)
\end{array}\right]
\end{gathered}
$$

Thus, taking $\left(\mathrm{F}_{k}^{v}, p_{k}^{v}\right)$ and $\left(\mathrm{F}_{k}^{x}, p_{k}^{x}\right)$ as the inputs of state equation $x(k+1)=f[x(k), v(k)]$, we can get the state prediction evidence.

4.3 Obtain observation prediction evidence $E_{k+1 \mid k}^{z}=\left(\mathbb{F}_{k+1 \mid k}^{z}, p_{k+1 \mid k}^{z}\right)$

Taking the state prediction evidence $\left(\mathrm{R}_{k+1 \mid k}^{\mathrm{x}}, \rho_{k+1 \mid k}^{x}\right)$ as the input of $g(x(k+1))$, we can get $E_{k+1 \mid k}^{z}=\left(\boldsymbol{F}_{k+1 \mid k}^{z}, p_{k+1 \mid k}^{z}\right)$ based on the EP in (18).

4.4 Get new evidence $\left(\hat{\mathbb{R}}_{k+1}^{x}, \hat{\rho}_{k+1}^{x}\right)$ to fuse observation prediction evidence $\left(\mathbb{F}_{k+1 \mid k}^{z}, p_{k+1 \mid k}^{z}\right)$ and observation evidence $\left(\mathrm{F}_{k+1}^{\mathrm{z}}, p_{k+1}^{z}\right)$ through ER rule.

The observation evidence $\left(\mathrm{F}_{k+1}^{z}, p_{k+1}^{z}\right)$ can be constructed by adding noise evidence $\left(\mathrm{F}_{k}^{w}, p_{k}^{w}\right)$ to $z_{k+1}$

$$
\begin{aligned}
& \mathrm{F}_{k+1}^{\lambda}=\left\{\left[\pi_{\alpha_{0}}^{w-}+\lambda_{k+1}, \pi_{\alpha_{0}}^{w+}+\lambda_{k+1}\right],\left[\pi_{a_{1}}^{w-}+\lambda_{k+1}, \pi_{a_{1}}^{w+}+\lambda_{k+1}\right],\right. \\
&\left.\cdots,\left[\pi_{\alpha_{q-1}}^{w-}+\lambda_{k+1}, \pi_{\alpha_{q-1}}^{w+}+\lambda_{k+1}\right], \Theta\right\} ; \quad p_{k+1}^{\lambda}=p_{k}^{w} \\
& \mathrm{~F}_{k+2}^{\lambda}=\left\{\left[\pi_{\alpha_{0}}^{w-}+\lambda_{k+2}, \pi_{\alpha_{0}}^{w+}+\lambda_{k+2}\right],\left[\pi_{a_{1}}^{w-}+\lambda_{k+2}, \pi_{a_{1}}^{w+}+\lambda_{k+2}\right],\right. \\
&\left.\cdots,\left[\pi_{\alpha_{q-1}}^{w-}+\lambda_{k+2}, \pi_{\alpha_{q-1}}^{w+}+\lambda_{k+2}\right], \Theta\right\} ; \quad p_{k+2}^{\lambda}=p_{k}^{w} \\
&\left(\mathrm{~F}_{k+1}^{\mathrm{z}}, p_{k+1}^{z}\right)=\left[\begin{array}{c}
\left(\mathrm{F}_{k+1}^{\lambda}, p_{k+1}^{\lambda}\right) \\
\left(\mathrm{F}_{k+2}^{\lambda}, p_{k+2}^{\lambda}\right)
\end{array}\right]
\end{aligned}
$$

Since $\left(\mathrm{F}_{k+1}^{\mathrm{z}}, p_{k+1}^{z}\right)$ and $\left(\mathrm{F}_{k+1 \mid k}^{\mathrm{z}}, p_{k+1 \mid k}^{z}\right)$ come from sensor and observation equation respectively, which are two independent information sources, so both of them can be fused using ER rule with weight and reliability. So we get the fused result $\hat{E}_{k+1}^{Z}=\left(\hat{F}_{k+1}^{Z}, \hat{p}_{k+1}^{Z}\right)$ to fuse $\left(\mathbb{F}_{k+1 \mid k}^{z}, p_{k+1 \mid k}^{z}\right)$ and $\left(\mathrm{F}_{k+1}^{z}, p_{k+1}^{z}\right)$ in observation domain. And new evidence $\left(\hat{\mathbb{R}_{k+1}^{x}}, \hat{\rho}_{k+1}^{x}\right)$ can be calculated using inverse function $g^{-1}\left(\mathrm{z}_{k+1}\right)$ and extension principles in state domain.

4.5 Get state estimation evidence $\left(\hat{\mathbb{F}}_{k+1 \mid k+1}^{x}, p_{k+1 \mid k+1}^{x}\right)$ to fuse new evidence $\left(\hat{\mathbb{R}_{k+1}^{x}}, \hat{\rho}_{k+1}^{x}\right)$ and state prediction evidence $\left(\mathrm{R}_{k+1 \mid k}^{x}, \rho_{k+1 \mid k}^{x}\right)$ through dependent evidence fusion

$\left(\hat{\mathbb{F}}_{k+1 \mid k+1}^{x}, p_{k+1 \mid k+1}^{x}\right)$ can be obtained by fusing new evidence $\left(\hat{\mathbb{R}}_{k+1}^{x}, \hat{\rho}_{k+1}^{x}\right)$ and state prediction evidence $\left(\mathrm{R}_{k+1 \mid k}^{x}, \rho_{k+1 \mid k}^{x}\right)$. However, since the new evidence $\left(\hat{\mathbb{R}}_{k+1}^{x}, \hat{\rho}_{k+1}^{x}\right)$ is obtained by observation prediction evidence $\left(\mathbb{F}_{k+1 \mid k}^{z}, p_{k+1 \mid k}^{z}\right)$ and observation evidence $\left(\mathrm{F}_{k+1}^{z}, p_{k+1}^{z}\right)$, and $\left(\mathbb{F}_{k+1 \mid k}^{z}, p_{k+1 \mid k}^{z}\right)$ is related to $\left(\mathrm{R}_{k+1 \mid k}^{x}, \rho_{k+1 \mid k}^{x}\right)$ in step 3), so $\left(\hat{\mathbb{R}_{k+1}^{x}}, \hat{\rho}_{k+1}^{x}\right)$ and $\left(\mathrm{R}_{k+1 \mid k}^{x}, \rho_{k+1 \mid k}^{x}\right)$ are also dependent on each other. Those two dependent evidences can be fused using independent evidence fusion theory in section 3.2 . 
The state estimation value $x_{k+1 \mid k+1}$ can be obtained by calculating pignistic expectation of $\left(\hat{\mathbb{F}}_{k+1 \mid k+1}^{x}, p_{k+1 \mid k+1}^{x}\right)$. The wavelength of each step can be estimated through iteration.

\section{Parameter training method of evidence reasoning fusion model}

In section IV, the initial parameter set $P=\left\{w_{o p}^{z}, w_{o}^{z}\right\}$ is used in the state estimation process, and finally the level estimation value is obtained. $w_{o p}^{z}$ and $w_{o p}^{z}$ represent the weight parameter set to be optimized for observation evidence and observation prediction evidence respectively. However, the ER reasoning model constructed from the initial parameter set cannot accurately obtain the final liquid level estimate. Therefore, the objective function of the parameter optimization model is

$$
\begin{aligned}
\min \xi(P) & =\frac{1}{S} \sum_{S=1}^{S}\left(L_{s}-\hat{L}_{s}\right)^{2} \\
0 & \leq w_{o p}^{z}, w_{o}^{z} \leq 1
\end{aligned}
$$

Where $L$ represents the true length, $\hat{L}$ represents the estimated value. The optimization process can be achieved by using gradient-based methods or nonlinear optimization software packages.

\section{Liquid Level Estimation Tests and Comparative analysis}

\subsection{Liquid level estimation tests}

The experimental equipment is shown as figure 3. The audio signal is generated by the audio card of the embedded controller, and the sound wave is emitted by the driver speaker.The sound frequency increases from $f_{1}=1000 \mathrm{~Hz}$ to $f_{\mathrm{h}}=2500 \mathrm{~Hz}$ at a constant speed within 5 seconds. The composite wave is captured by the microphone and further processed by the controller (STM32F756NG). In the experiment, the detection height $L=1.6 \mathrm{~m}$, the sound velocity $c=347.3 \mathrm{~m} / \mathrm{s}$, and the temperature $T=26.5^{\circ} \mathrm{C}$.

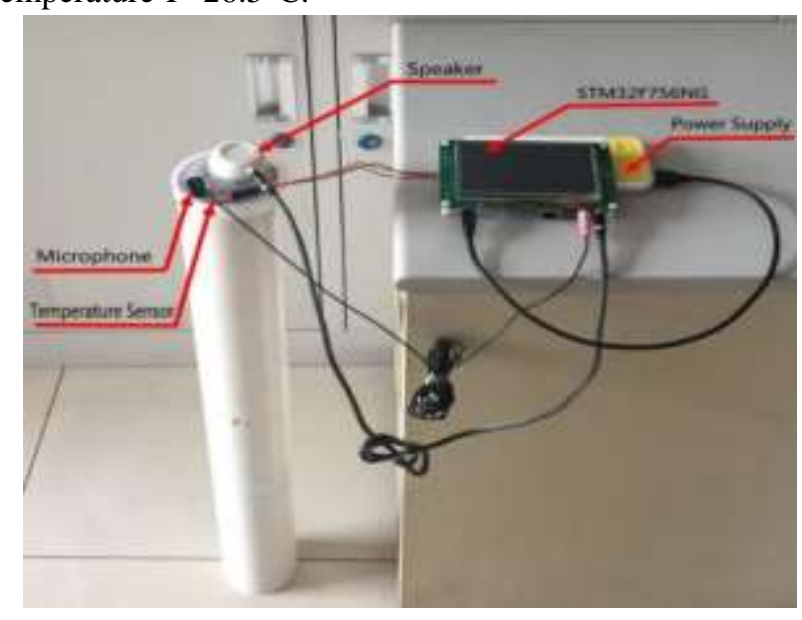

Fig 3: Experiment set-up

The synthesized sound wave collected by the microphone is shown in Figure 4, and 14 resonance frequency points are generated in total. Then, the frequency and wavelength corresponding to the resonance frequency point are extracted in the amplitude detection through fast Fourier transform and smoothing filter ${ }^{[8,9]}$, as shown in Figure 5. Obviously, because the synthesized sound wave is influenced by external noise and sound wave attenuation, the missed detection phenomenon occurs in the extraction process of resonance frequency point. And 13 resonance

ISSN: 0010-8189 
frequency points are extracted, and the information of 7th resonance frequency point is not extracted.The missed resonance point will seriously reduce the accuracy in the subsequent liquid level calculation.

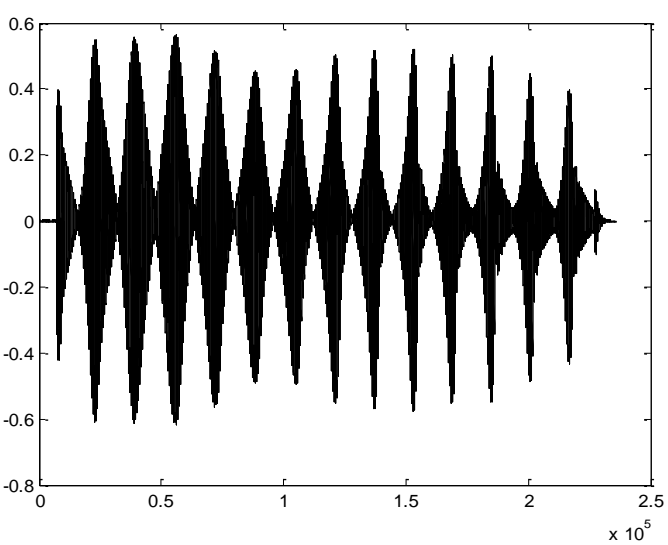

Fig 4: Waveform graph $(L=1.6 m)$

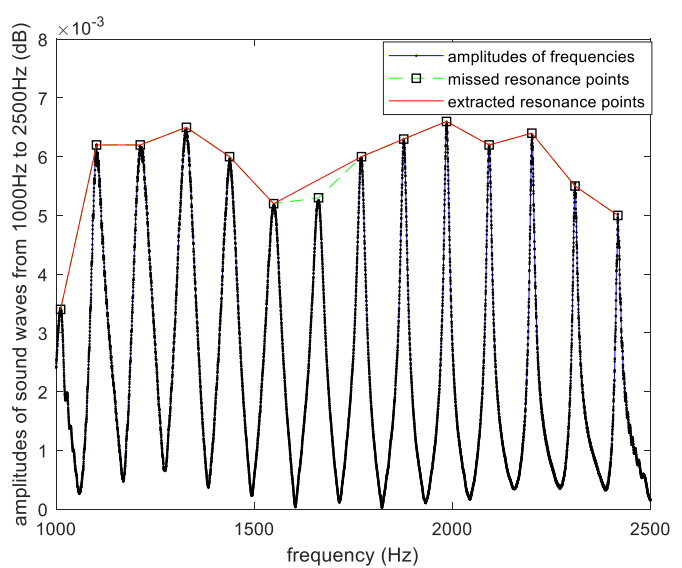

Fig 5: Resonance frequencies $(L=1.6 m)$

The state/observation noise $(v / w)$ is approximated to triangle possibility distribution respectively ${ }^{[10]}$. In order to construct state noise evidence,we use a high-precision oscilloscope to collect the synthetic sound waves emitted by the sound-producing equipment (speakers) in a fixed frequency range, and select and calculate the error of 100 frequency points. The value range of the state noise is calculated to be plus or minus three times the standard deviation of the error. The evidence can be structured as shown in Table 1.

The value range of observation noise depends on the sound receiving equipment, smoothing filter and Fourier transform algorithm. We collect 42 sets of sample data in the range $L=0.6 \mathrm{~m}$ to $L=10.8 \mathrm{~m}$, and use fast Fourier transform and smoothing filtering algorithm to extract the wavelength observation value corresponding to the standing wave.Then, according to the error between theoretical value (true value) and observed value, observation noise evidence $\left(\mathrm{F}_{k}{ }^{w}, p_{k}^{w}\right)$ can be structured as shown in Table 2.

Table 1 Evidence of $v(k)$

\begin{tabular}{|l|c|c|c|}
\hline $\mathrm{F}_{k}^{v}$ & {$[-0.0043,0.0043]$} & {$[-0.0029,0.0029]$} & {$[-0.0015,0.0015]$} \\
\hline$p_{k}^{v}$ & $1 / 3$ & $1 / 3$ & $1 / 3$ \\
\hline
\end{tabular}

Table 2 Evidence of $w(k)$

\begin{tabular}{|l|c|c|c|}
\hline $\mathrm{F}_{k}^{w}$ & {$[-0.0433,0.0589]$} & {$[-0.0263,0.0419]$} & {$[-0.0093,0.0249]$} \\
\hline$p_{k}^{w}$ & $1 / 3$ & $1 / 3$ & $1 / 3$ \\
\hline
\end{tabular}

From figure 4 and formula (5), we can calculate the first and second standing wave wavelength $\left(\lambda_{1}=0.3433 \mathrm{~m}\right.$ and $\left.\lambda_{2}=0.3151 \mathrm{~m}\right)$ respectively. The initial observation value is $z(1)^{T}=[0.3433,0.3151]$, the first estimation result $\hat{x}_{1 \mid 1}^{T}=z(1)^{T}$. After constructing state noise evidence $\left(\mathrm{F}_{1}^{v}, p_{1}^{v}\right)$ and observation noise evidence $\left(\mathrm{F}_{1}{ }^{w}, p_{1}^{w}\right)$, according to the iterative steps in section IV, we can obtain $\left(F_{k+1 k}^{z}, p_{k+1 \mid k}^{z}\right)$ and $\left(\mathrm{F}_{k+1}^{z}, p_{k+1}^{z}\right)$, and use the ER rule to fuse those two evidence with weight and reliability in observation domain. Then the fused new evidence $\left(\hat{\mathbb{R}}_{k+1}^{x}, \hat{\rho}_{k+1}^{x}\right)$ and state evidence $\left(\mathrm{R}_{k+1 \mid k}^{x}, \rho_{k+1 \mid k}^{x}\right)$ are combined with evidence independence in state domain. Finally, the wavelength value at each time can be estimated through the iterative algorithm, and the corresponding liquid level height can be 
calculated by formula (4). In the estimation process, we set $r_{o p}^{\mathrm{z}}=0.8, r_{o}^{\mathrm{z}}=0.3$ according to experience, and adjust optimized parameter $w_{o p}^{z}=0.9862, w_{o}^{z}=0.2015$ according to parameter training method.

\subsection{Comparative analysis}

In order to further verify the validity and feasibility of this method, figure 6 gives estimation results of the direct observation method, the Nasreddine's method and the proposed method. Obviously, there is missed resonance point $(\lambda(7)=0.2091 \mathrm{~m})$ in the process of extracting resonance points, which makes the observation method lack a calculation result $(L(7)=1.6042 \mathrm{~m})$ and seriously reduces the reliability and accuracy of the final result. The proposed method uses the two-dimensional system dynamic model about the standing wave wavelength, the standing wave wavelength of the next moment can be estimated accurately according to the information of the two adjacent moments. Our method can effectively overcome the missed detection phenomenon and improve the antiinterference ability and accuracy of the acoustic resonance liquid level detection method.

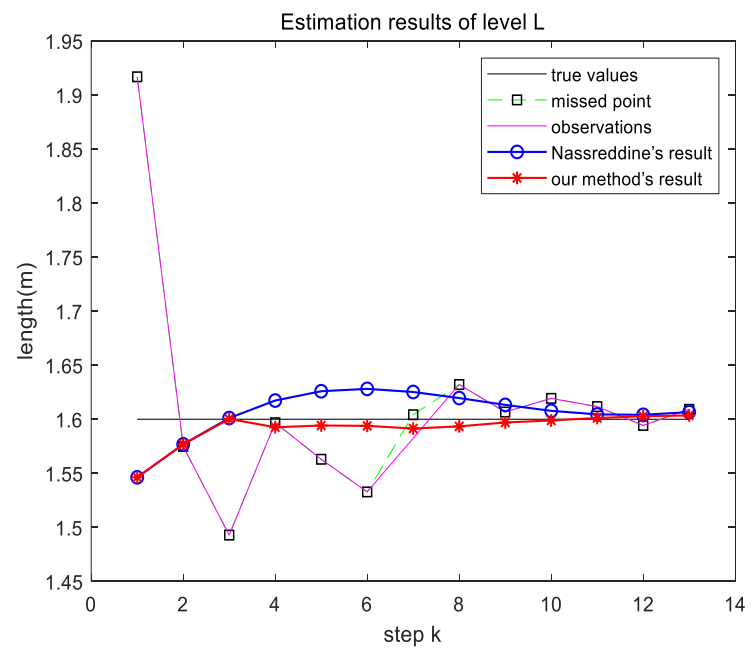

Fig6: Estimation results

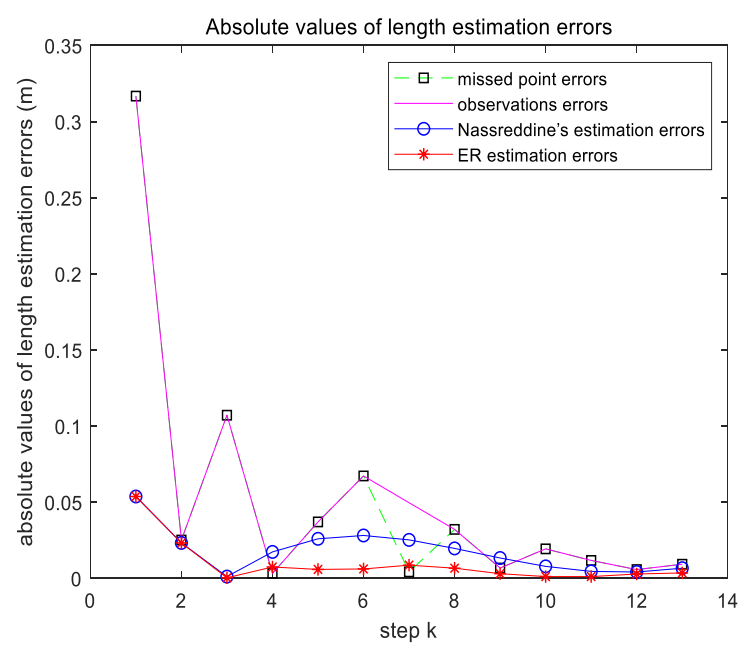

Fig 7: Absolute values of estimation errors

Figure 7 shows the absolute values of length estimation errors. The proposed method considering bounded noise can effectively suppress and reduce the influence of system noise on the liquid level estimation process. The dependent evidence fusion considering evidence relevance can make the estimation result more focused on the real

ISSN: 0010-8189 
state. Table 3 lists more experimental results of different lengths, which further illustrate the validity and feasibility of this method.

Table 3 More experimental results of different lengths

\begin{tabular}{|l|c|c|c|}
\hline Different methods & True $L(\mathrm{~m})$ & Err $(\mathrm{m})$ & Precision \\
\hline Direct measurement & 0.5 & 0.0220 & $2.400 \%$ \\
Nassreddine method & & 0.0128 & $1.360 \%$ \\
Our method & & 0.0068 & $2.875 \%$ \\
\hline Direct measurement & 1.6 & 0.0460 & $0.975 \%$ \\
Nassreddine method & & 0.0156 & $0.693 \%$ \\
Our method & 2.1 & 0.0111 & $2.152 \%$ \\
\hline Direct measurement & & 0.0452 & $0.742 \%$ \\
Nassreddine method & & 0.0156 & $0.371 \%$ \\
Our method & 3.6 & 0.0078 & $0.919 \%$ \\
\hline Direct measurement & & 0.0331 & $0.358 \%$ \\
Nassreddine method & & 0.0129 & $0.275 \%$ \\
Our method & 5.7 & 0.0099 & $1.200 \%$ \\
\hline Direct measurement & & 0.0684 & $0.154 \%$ \\
Nassreddine method & & 0.0088 & $0.117 \%$ \\
Our method & 6.4 & 0.0067 & $1.375 \%$ \\
\hline Direct measurement & & 0.0880 & $0.217 \%$ \\
Nassreddine method & & 0.0139 & $0.098 \%$ \\
Our method & 7.5 & 0.0063 & $1.413 \%$ \\
\hline Direct measurement & & 0.1060 & $0.240 \%$ \\
Nassreddine method & & 0.0180 & $0.092 \%$ \\
Our method & & 0.0069 & \\
\hline
\end{tabular}

\section{Conclusion}

This paper presents a novel acoustic fluid level estimation method based on evidence fusion mechanism. This method can deal with the missed detection error and the systematic error caused by acoustic resonance fluid level detection. The proposed method establishes a two dimensional dynamic system model based on the relationship between any three adjacent resonance points, and models state noise and observation noise of systematic error as the triangular possibility distribution respectively, and then constructs the state evidence and observation evidence by adding those noise via the random set description. The recursive algorithm based on ER rule and dependent evidence fusion is proposed to estimate the wavelength of resonance point at each moment. The detected height can be calculated from the fused result based on Pignistic expectation. Compared with other algorithm, our method overcome the missed detection phenomenon and improve the anti-interference ability and accuracy of the acoustic resonance liquid level detection. However, there are some further work to do for enhancing the accuracy of the proposed method. For examples, (1) how to further improve the accuracy of the optimization algorithm for the parameter set, (2) how to combine error compensation method with state estimation method to further improve accuracy and reliability. In the future, the research on those problems should be further studied.

\section{Acknowledgements}

This research was funded by NSFC (No: 62003313, 6200300, 61803086) and Key scientific and technological projects in Henan Province (202102210125, 202102210329, 21B510013).

\section{References}

[1] L. Wang, Z. J. Zhou, C. H. Hu, et al, “ Ultrasonic testing defect recognition based on belief-rule-base and evidential reasoning,” China Meas. Test, vol. 43, no. 4, pp. 62-67, 2017.

[2] Donlagic, D. M. Zavrsnik, I. Sirotic, "The use of one-dimensional acoustical gas resonator for fluid level measurements," IEEE Tran on Instrumentation and Measurement, Vol. 49, No 5, pp. 1095-1110,

ISSN: 0010-8189

C CONVERTER 2020

Www.converter-magazine.info 
2000 .

[3] X.-B. Xu, C.-P. Zhao, B.-D. Xia, "A fluid level measurement method based on acoustical resonance in a special frequency range”, Acta Metrol Sinica, vol. 32, no. 1, pp. 53-57, 2011.

[4] X. Xu, Z. Li, G. Li, et al., "An Acoustic Resonance-Based Liquid Level Detector With Error Compensation”, IEEE Transactions on Instrumentation and Measurement, vol. 68, no. 4, pp. 963-971, 2019.

[5] J. B. Yang, D. L. Xu, "Evidential reasoning rule for evidence combination,”.Artificial Intelligence, vol. 205, pp. 1-29, 2013.

[6] J. B.Yang, D. L. Xu, "On the evidential reasoning algorithm for multiattribute decision analysis under uncertainty," IEEE Transactions on Systems, Man, and Cybernetics Part A: Systems and Humans, vol. 32, no. 3, pp. 289-304, 2002.

[7] H. Lü, W.-B. Shangguan, D. Yu, "An imprecise probability approach for squeal instability analysis based on evidence theory," J. Sound Vibrat,vol. 387, pp. 96-113, 2017.

[8] X Xu, Z Li, G Li, et al., "State Estimation Using Dependent Evidence Fusion: Application to Acoustic Resonance-Based Liquid Level Measurement," Sensors, no. 4: 924, 2017.

[9] D. P. Bertsekas, I. B. Rhodes, "Recursive state estimation for a set-membership description of uncertainty," IEEE Trans. Autom. Control, vol. AC-16, no. 2, pp. 117-128, Apr 1971.

[10] G. Nassreddine, F. Abdallah, T. Denoux, "State estimation using interval analysis and belief-function theory: application to dynamic vehicle localization,” IEEE Trans. Syst., Man, Cybern. B, Cybern. vol. 40, no.5, pp. 1205-1218, Oct 2010. 